\title{
Will the COVID-19 pandemic worsen the obesity epidemic?
}

\section{Christoffer Clemmensen $\mathbb{1}^{1 凶}{ }^{凶}$, Michael Bang Petersen ${ }^{2}$ and Thorkild I. A. Sørensen ${ }^{1,3}$}

The sudden outbreak and global spread of COVID-19 represents one of the most profound societal and public health challenges in modern times. In this Comment, we call attention to the possibility that the societal strategies implemented to oppose COVID-19 might have long-term, negative effects on the obesity epidemic.

\begin{abstract}
A key measure used worldwide to combat the COVID-19 pandemic has been the rapid and strict enforcement of physical distancing. To facilitate this measure, governments have imposed pervasive societal interventions, such as lockdowns, in the public and private sectors. While the intended consequence was to isolate millions of individuals, thereby containing viral transmission, an unintended consequence has been an economic crisis as companies are pushed into bankruptcy and unemployment rates increase rapdily. Here, we discuss the possibility that the combination of imposed isolation and subsequent socioeconomic hardship and deterioration of psychosocial health might result in long-lasting effects on metabolic health. In societies with obesogenic environments, this effect might worsen rates of obesity and obesity-linked metabolic comorbidities (FIG. 1).
\end{abstract}

\section{Increased socioeconomic hardship}

In many countries, the interventions required to combat COVID-19 have resulted in a severe socioeconomic crisis. In countries with liberal market economies and a lack of solidarity-based welfare programmes, socioeconomic challenges often worsen the circumstances for the poorest people in society and thereby exacerbate socioeconomic inequality and polarization of population segments, as seen following the financial crisis of 2007-2008 (REF. ${ }^{1}$ ).

Given the well-described relationship between socioeconomic status and risk of obesity, a widening societal inequality propelled by the political interventions against COVID-19 might translate into an increase in obesity occurrence and metabolic diseases in groups with a lower socieconomic status. One explanation for this increase is that an abundance of highly processed, energy-rich, palatable, cheap and readily available foods promotes calorie intake beyond energetic needs ${ }^{2}$, and such foods are often preferentially selected by individuals with a lower socieconomic status who have limited income and resources ${ }^{3}$.

\section{Deteriorating psychosocial health}

The existence of a global pandemic in and of itself can create psychological stress ${ }^{4}$, but feelings of anxiety might be exacerbated by a worsening of socioeconomic conditions. Moreover, physical distancing might remove the key medium for psychological comfort for many individuals, that is, meaningful social interaction, and increase feelings of loneliness. As such, the COVID-19 pandemic offers a 'perfect storm' for inducing psychosocial insecurity.

Deteriorating psychosocial health can have a negative effect on food-related behaviour. The temporary, but cross-culturally consistent pattern of food hoarding is one example. More broadly, psychosocial stress reactions have been found to increase energy intake ${ }^{5}$, and individuals with limited social interactions are at an increased risk of developing obesity ${ }^{6}$. Home confinement during the COVID-19 pandemic provides an altered food cue exposure, which could challenge the individual's cognitive restraint and enhance impulsive eating behaviour. In addition, emotional eating, often used to relieve negative feelings, might increase under these circumstances. The elimination of social eating practices could encourage a reduction in mindful eating, which might negatively influence dietary choices and promote overeating ${ }^{7}$. Finally, the closing of fitness centres and curtailment of organized sports, in combination with the need for physical distancing, could make maintaining an active lifestyle difficult.

While lockdown-induced psychosocial insecurities might be temporally short-lived, even brief periods of energy excess can result in sustained negative effects on obesity and metabolic health ${ }^{8}$, probably owing to homeostatic forces rapidly adapting to defend elevated body adipose levels. More extreme temporary food insecurity can also impose long-lasting effects on eating behaviour and body weight, as seen in refugees?

\section{Perturbations in energy homeostasis}

Exactly how socioeconomic status and psychosocial health interact with the biological systems governing weight homeostasis is an open question. Physiological 


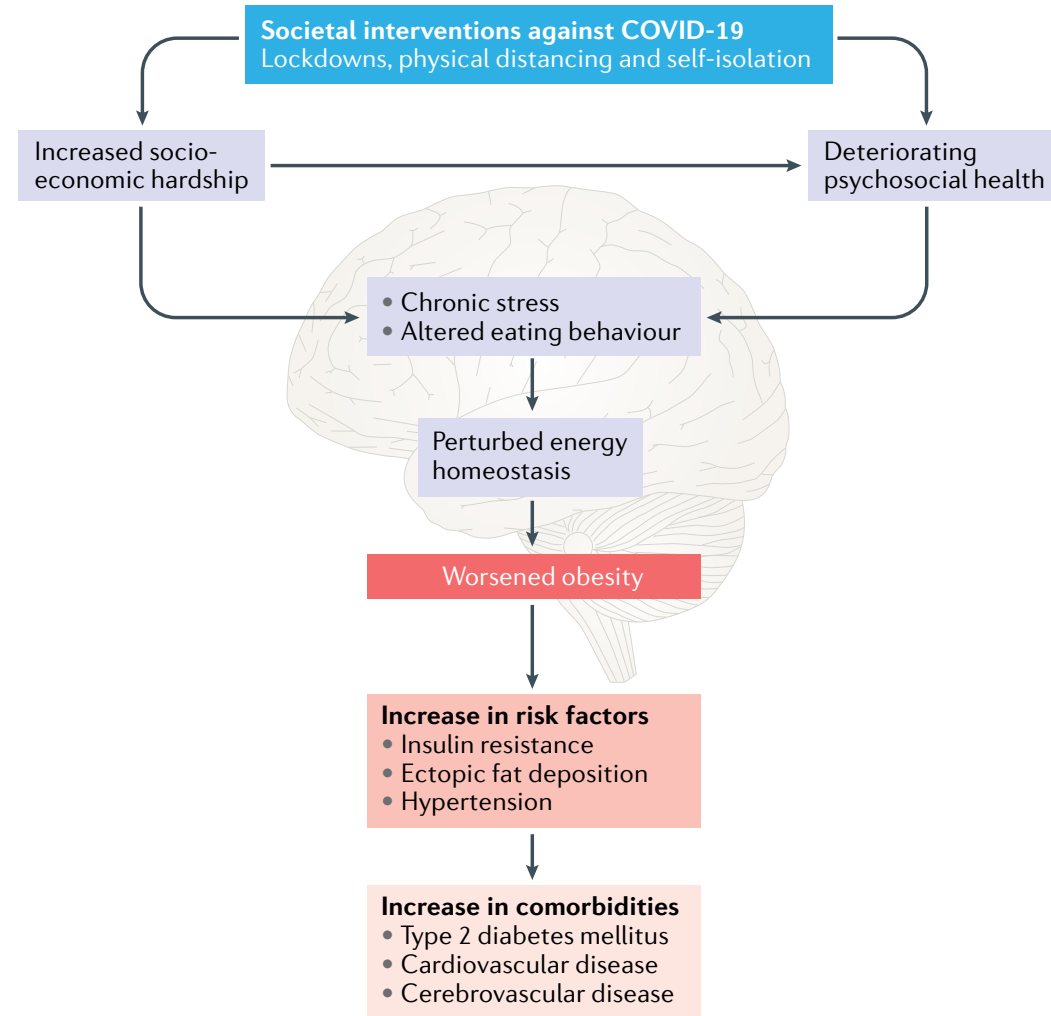

Fig. 1 Proposed mechanisms by which the societal strategies used to combat COVID-19 might worsen the occurrence of metabolic diseases. Societal interventions against COVID-19 might trigger a series of psychobiological mechanisms that accelerate obesity occurrence and increase the risk of developing obesity-linked comorbidities.

stress might be a gateway by which psychosocial conditions affect neuroendocrine systems regulating energy metabolism. Brain structures, including the cortex, amygdala and hippocampus, perceive and respond to threats to systemic homeostasis, including emotional distress. The neural pathways converge in the paraventricular nucleus, which helps modulate the sympathetic nervous system and the hypothalamic-pituitary-adrenal axis ${ }^{10}$. A stress-induced increase in circulating levels of glucocorticoids is linked to consumption of palatable foods and uncoupling of energy-need based eating ${ }^{10}$. Excess energy intake, without a concomitant increase in substrate metabolism and/or fat storage capacity, might accelerate lipotoxicity, ectopic fat deposition, low-grade inflammation and insulin resistance. These pathogenic processes can be further exacerbated by physical inactivity.

\section{Conclusions}

The use of lockdowns to combat the COVID-19 pandemic seems to have been successful from an epidemiological perspective, but lockdowns might have serious negative consequences on other health metrics.
Specifically, lockdowns might adversely affect metabolic health via combined deterioration of socioeconomic conditions, psychological security and metabolic processes. To this end, approaches designed to contain the spread of COVID-19 might promote obesity and associated metabolic diseases.

If the prediction of a worsening of the obesity epidemic turns out to be true, a major task lies in dissecting the underlying causal pathways. Understanding how the strategies used to combat COVID-19 worsen metabolic health is necessary for designing meaningful counter-strategies. Eventually, such insights should guide governments and policy makers to implement individual and/or societal interventions toward containing lockdown-linked worsening of obesity and its metabolic comorbidites. Given our review of the potential pathways, however, we expect an emphasis on socioeconomic safety nets and community support networks to be key. One challenge is that it might be difficult to build the required support structures after the fact, and it might be difficult to fully contain the potential obesity-related effects of a lockdown. Accordingly, when considering the use of lockdowns in the future, the potential adverse consequence on metabolic health should be taken into account.

1. Iversen, T. \& Soskice, D. Democracy and prosperity: Reinventing capitalism through a turbulent century (Princeton University Press, 2019).

2. Schwartz, M. W. et al. Obesity pathogenesis: an Endocrine Society scientific statement. Endocr. Rev. 38, 267-296 (2017).

3. Darmon, N. \& Drewnowski, A. Does social class predict diet quality? Am. J. Clin. Nutr. 87, 1107-1117 (2008).

4. World Health Organization. Mental health and psychosocial considerations during the COVID-19 outbreak. WHO https://www. who.int/publications/i/item/WHO-2019-nCoV-MentalHealth-2020.1 (2020).

5. Bjorntorp, P. Do stress reactions cause abdominal obesity and comorbidities? Obes. Rev. 2, 73-86 (2001).

6. Kim, D., Subramanian, S. V., Gortmaker, S. L. \& Kawachi, I. US state- and county-level social capital in relation to obesity and physical inactivity: a multilevel, multivariable analysis. Soc. Sci. Med. 63, 1045-1059 (2006).

7. Higgs, S. \& Thomas, J Social influences on eating. Curr. Opin. Behav. Sci. 9, 1-6 (2016).

8. Yanovski, J. A. et al. A prospective study of holiday weight gain N. Engl. J. Med. 342, 861-867 (2000)

9. Peterman, J. N. et al. Relationship between past food deprivation and current dietary practices and weight status among Cambodian refugee women in Lowell, MA. Am. J. Public Health 100, 1930-1937 (2010).

10. Ulrich-Lai, Y. M. \& Ryan, K. K. Neuroendocrine circuits governing energy balance and stress regulation: functional overlap and therapeutic implications. Cell Metab. 19, 910-925 (2014).

\section{Acknowledgements}

C.C. receives funding from the Lundbeck Foundation (Fellowship R238 2016-2859) and the Novo Nordisk Foundation (grant number NNF17OC0026114). M.B.P. receives funding the Carlsberg Foundation (grant number CF20-0044). Novo Nordisk Foundation Center for Basic Metabolic Research is an independent Research Center based at the University of Copenhagen, Denmark, and partially funded by an unconditional donation from the Novo Nordisk Foundation (www.cbmr.ku.dk) (Grant number NNF18CC0034900).

\section{Competing interests}

The authors declare no competing interests. 